\title{
OXIDACIÓN CATALÍTICA SELECTIVA PARA LA CONVERSIÓN DE METANO A METANOL: UNA REVISIÓN*
}

\section{SELECTIVE CATALYTIC OXIDATION FOR METHANE CONVERSION TO METHANOL: A REVIEW}

William Giovanni Cortés-Ortiz*

Carlos Alberto Guerrero-Fajardo"

Fecha de recepción: 2 de diciembre de 2016

Fecha de revisión: 29 de mayo de 2017

Fecha de aprobación: 4 de julio de 2017

Cómo citar: W. G. Cortés-Ortiz y C. A. Guerrero-Fajardo, "Oxidación catalítica selectiva para la conversión de metano a metanol: Una revisión," Ciencia e Ingeniería Neogranadina, vol. 28, no. 1, pp. 45-71. DOI: https://doi. org/10.18359/rcin.2623

\section{RESUMEN}

La oxidación de metano a metanol por medio de una forma directa, económica y con bajo gasto energético es un objetivo perseguido por la industria desde sus orígenes. El metano es el principal componente del gas natural, mientras que el metanol es combustible y materia prima en la industria química. En el presente artículo de revisión se presentan los resultados de trabajos realizados para lograr dicho proceso, identificando los aspectos más relevantes que se involucran. Se encontraron bajos valores de conversión de metano y selectividad a metanol debido, principalmente, a dos factores: el primero es la dificultad de activar el metano y, el segundo, poder controlar el proceso para evitar reacciones secuenciales de oxidación que generen otros productos. Para incrementar el rendimiento del proceso, es necesario optimizar las condiciones de síntesis de los materiales, incluyendo temperatura, tiempo y cargas del componente activo, lo cual influirá en el comportamiento físico y químico de los catalizadores. También es necesario controlar las variables del proceso de oxidación catalítica, como el tipo de material del reactor,

* $\quad$ Licenciado en Química; magíster en Ciencias Ambientales y doctor (c) en Ingeniería de Materiales, Universidad Nacional de Colombia. Profesor ad-honorem, Universidad El Bosque, Bogotá, Colombia. Correo electrónico: wgcorteso@unal.edu.co. ORCID: https://orcid.org/0000-0003-4714-9104

** Químico, Ingeniero Mecánico, MSc. y Ph.D. en Química. Profesor Asociado, Universidad Nacional de Colombia, Bogotá. Correo electrónico: caguerrerofa@unal.edu.co. ORCID: https://orcid.org/0000-0002-9844-4114 
flujos de alimentación, tiempos de permanencia de los gases dentro del reactor, y evitar así reacciones competitivas que disminuyan la selectividad al alcohol.

Palabras clave: metano, metanol, oxidación selectiva, catalizadores sólidos.

\section{ABSTRACT}

The oxidation of methane to methanol using a direct, economical and low energy cost is an objective pursued by the industry since its inception. Methane is the main component of natural gas, while methanol is both fuel and raw material in the chemical industry. This review article presents the results of work done to achieve this process, identifying the most relevant aspects involved. Low values of methane conversion and selectivity to methanol were found mainly due to two factors: the first is the difficulty of activating methane and the second, being able to control the process to avoid sequential oxidation reactions that generate other products. To increase the performance of the process is necessary to optimize the synthesis conditions of the materials including temperature, time and loads of the active component, which will influence the physical and chemical behavior of the catalyst. It is also necessary to control the variables of the catalytic oxidation process, such as the type of reactor material, feed flows, the residence times of the gases inside the reactor, and thus avoid competitive reactions that decrease the selectivity to the alcohol.

Keywords: Methane, methanol, selective oxidation, solid catalysts.

\section{INTRODUCCIÓN}

La oxidación catalítica de metano para la producción de metanol o formaldehído ha captado la atención de los investigadores desde el inicio de la catálisis. El metano es el principal constituyente del gas natural, mientras que el metanol es empleado como combustible y como materia prima en la industria química. En las décadas recientes, el mecanismo para la oxidación parcial de metano a metanol o formaldehído sobre catalizadores de óxido de metal ha sido tema de interés de muchos investigadores [1]. Sin embargo, a pesar de los extensos estudios teóricos y experimentales, los procesos químicos implicados no han sido totalmente comprendidos y todavía dan lugar a controversia.

Algunos estudios sugieren que el metano se oxida directamente a formaldehído y metanol y luego a $\mathrm{CO}$ y el $\mathrm{CO}_{2}$ mediante reacciones consecutivas [2]. Otros autores proponen la existencia de un proceso paralelo consecutivo [3], mientras que algunos sugieren que la oxidación de metano sobre catalizadores de PdO sigue un mecanismo de oxidación reducción de tipo MarsVan Krevelen [4].

En este artículo se presentan diferentes resultados de trabajos realizados para lograr la oxidación directa de metano a metanol, y 
se ha dividido en cuatro secciones: 1) generalidades de metano; 2) producción de metanol; 3) resultados de las investigaciones publicadas sobre el proceso de oxidación directa de metano, y 4) conclusiones. Cabe destacar que se han reportado valores bajos de conversión de metano y selectividad de metanol para ser considerado un proceso viable industrialmente, lo cual genera una amplia oportunidad para estandarizar las técnicas de síntesis de catalizadores, así como las variables del proceso buscando obtener valores altos de rendimiento.

\section{METANO}

El gas natural está compuesto principalmente por metano, con reservas probadas de gas natural que actualmente se estima en 186,9 trillones de metros cúbicos (tcm), cantidad suficiente para cubrir la producción actual durante 52,8 años [5].
Como los combustibles fósiles, el gas natural es raramente puro; es comúnmente asociado con petróleo y a menudo contiene otros hidrocarburos, incluyendo etano, butano y propano. El gas natural es un combustible esencial para la sociedad moderna, pues durante los últimos cincuenta años del siglo XX proporcionó aproximadamente el $25 \%$ de la demanda de energía de Estados Unidos y se ha utilizado para la generación de energía eléctrica, procesos de calor industrial, calefacción, cocina doméstica y como combustible para el transporte [6].

Las reacciones principales en las cuales interviene el metano se presentan en la Tabla 1. Se destaca que las oxidaciones parciales son difíciles de controlar y normalmente ocurren de manera completa, y esto genera como producto dióxido de carbono y agua.

Tabla 1. Reacciones de metano

\begin{tabular}{|l|l|}
\hline \multicolumn{1}{|c|}{ Reacción } & \multicolumn{1}{c|}{ Consideración } \\
\hline Oxidación & $\begin{array}{l}\text { Los productos dependen de la concentración de } \\
\text { los reactivos y las condiciones del proceso como } \\
\text { temperatura o presión. }\end{array}$ \\
\hline Reacciones ácido-base & $\begin{array}{l}\text { En mezclas de gas a baja presión una variedad de iones } \\
\text { positivos derivados de metano ha sido observada: catión } \\
\text { metilo } \mathrm{CH}^{+3} \text { y metano protonado } \mathrm{CH}^{+5}[7] .\end{array}$ \\
\hline Halógenos & $\begin{array}{l}\text { El mecanismo se denomina halogenación con radicales } \\
\text { libres. Se inicia con la luz UV o algún otro iniciador } \\
\text { radical [8]. }\end{array}$ \\
\hline
\end{tabular}

Fuente: elaboración propia. 
De acuerdo con lo anterior, una reacción de gran interés es la oxidación selectiva de metano a metanol, para la cual se han propuesto diferentes enfoques; sin embargo, hasta ahora ninguno es factible industrialmente, ya que el metanol es menos estable que los otros productos de oxidación. Por tal razón, desarrollar una ruta directa para producir metanol a partir de metano ha sido referido como un proceso muy complejo debido a su enorme dificultad [9].

\section{PRODUCCIÓN DE METANOL}

Robert Boyle descubrió el metanol en 1661, aunque no hay constancia escrita de cualquier uso doméstico o industrial antes del siglo XIX. En 1834, Dumas y Peligot establecieron por primera vez la identidad química y molecular del metanol. El primer método de producción de metanol fue la destilación destructiva de la madera; "espíritu de la madera" o "alcohol de madera" fue el nombre dado al metanol durante muchos años, refiriéndose a este método de síntesis. El químico francés Paul Sabatier aisló el metanol sintético por primera vez en 1905 y la fábrica badense de bicarbonato de sodio y anilina (BASF) comercializó la primera planta de metanol sintético en 1934 [10].

La producción de metanol en Estados Unidos se inició en 1927 por la Corporación Comercial de Solventes (CSC) y DuPont. El proceso llevado en CSC incluye la producción de $\mathrm{CO}_{2}$ y luego hidrogenarlo a metanol a 300 atmósferas empleando catalizadores de óxido de metal. El proceso de DuPont utiliza carbón para producir una materia prima gaseosa conocida como gas de síntesis, compuesta principalmente de monóxido de carbono e hidrógeno. El gas de síntesis se purifica y se pasa a través de un catalizador de síntesis de metanol, que es el producto final [11].

En 1966, la industria química imperial (ICI) presentó los óxidos de zinc y cobre como catalizadores que eran mucho más activos en la síntesis de metanol. La aplicación de estos catalizadores llevó a la ruta de síntesis de metanol por medio de alta presión; sin embargo, estos nuevos materiales catalíticos de óxido de cobre y zinc fueron altamente susceptibles al envenenamiento y la desactivación, lo cual generó la necesidad de realizar un estrecho control de los reactores para la producción de metanol [12].

La oxidación directa de metano a metanol es una ruta atractiva porque elimina un paso de mucha demanda de energía que actualmente es requerido para la producción de metanol; dicho paso es reformar el metano en gas de síntesis. Los estudios muestran que la producción de metanol a través de un proceso directo sería competitiva con respecto a los actuales métodos de producción de metanol si se logra al menos el 5,5\% de rendimiento con selectividades a metanol superiores al $80 \%$ [13].

Actualmente la industria del metanol abarca todo el mundo, con producción en Asia, América del Norte y del Sur, Europa, África y Oriente Medio. En todo el mundo, hay más de 90 plantas de metanol que tienen una capacidad de producción combinada de alrededor de 110 millones de toneladas métricas (casi 36,6 mil millones de galones o 138 mil millones de litros). La demanda mundial de metanol alcanzó los 70 millones de toneladas métricas en 2015, lo que impulsó en gran parte 
las aplicaciones energéticas emergentes del metanol, que ahora representan el $40 \%$ del consumo de este alcohol. Cada día, cerca de 200.000 toneladas de metanol se utilizan como materia prima química o como combustible de transporte [14].

\section{OXIDACIÓN SELECTIVA DE METANO}

En este apartado se presentan los resultados más relevantes acerca de la oxidación selectiva de metano a metanol indicando los tipos de catalizadores empleados, las condiciones del proceso, así como los valores de conversión de metano y selectividad a metanol. De la misma manera, se abordan las consideraciones físicas y químicas relacionadas con tales resultados.

\subsection{Metales y óxidos metálicos como catalizadores}

Un catalizador funcional para la oxidación parcial de metano a metanol atrajo intereses desde inicios del siglo $\mathrm{XX}$, particularmente con una de las primeras patentes concedidas a Lance y Elworthy [15] en 1905 , seguido por una patente británica en 1906 [16]. La patente de 1905 afirmaba que el formaldehído, ácido fórmico y el metanol podrían ser obtenidos por oxidación del metano con peróxido de hidrógeno en presencia de sulfato ferroso [15]. Asimismo, una serie de procesos a presión atmosférica fueron patentados al comienzo de 1900, como los de Gleck en los que el gas natural y el aire eran pasados por gránulos de piedra pómez o cobre a $800{ }^{\circ} \mathrm{C}$ [15].
Pruebas de nuevos trabajos en catalizadores para la oxidación parcial de metano a metanol no fueron visibles hasta 1921, cuando se hizo evidente que las posibilidades de producción de metanol a partir de gas natural fueron de considerable interés para el departamento canadiense de minas [17]. Un gran número de métodos para la producción de metanol y formaldehído fueron investigados (ver Fig. 1).

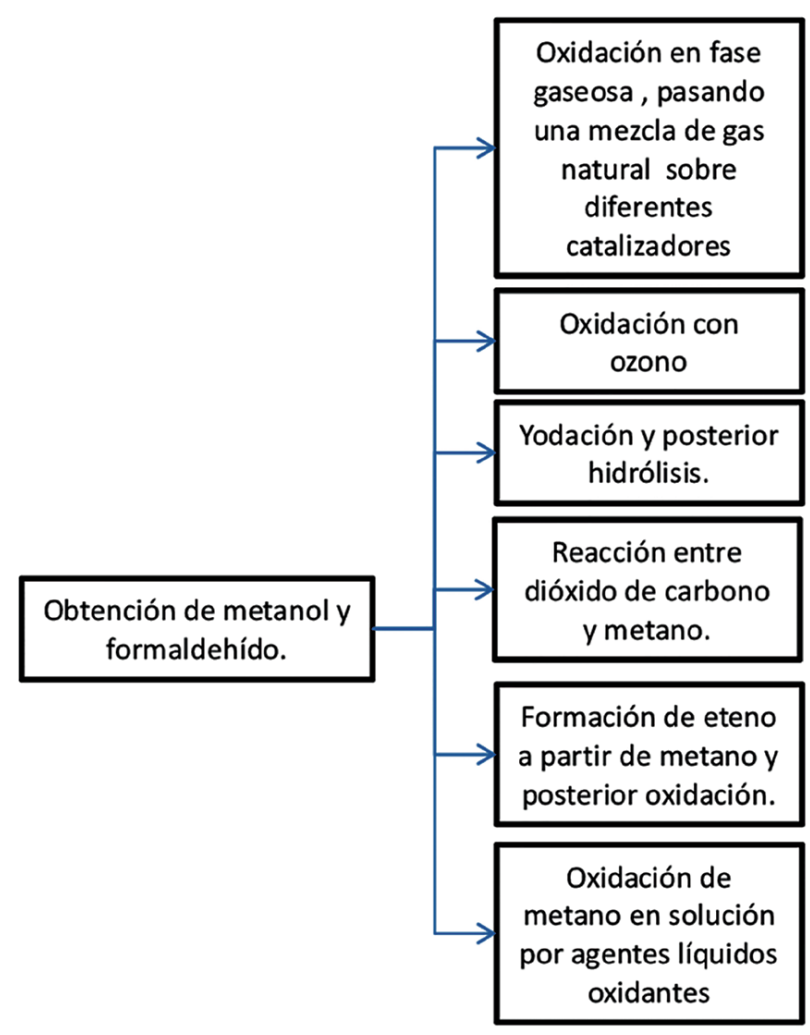

Fig. 1. Métodos para la producción de metanol y formaldehído

Fuente: elaboración propia.

De acuerdo con las diferentes propuestas presentadas en la Fig. 1, los rendimientos obtenidos eran demasiado pequeños para ser de importancia comercial; no obstante, 
la oxidación por ozono parecía ser el camino más prometedor [15].

En 1929 y 1930, se otorgaron patentes para el primer proceso de alta presión, en el cual una mezcla de gases que contienen gas natural y oxígeno, en igual volumen, se pasa por un catalizador a presiones entre 60 y 300 atm y a temperaturas entre 250 y $500{ }^{\circ} \mathrm{C}$. Los catalizadores contenían óxido de zinc y plomo u óxido de plomo. Los óxidos de níquel, cromo o talio también fueron propuestos como promotores útiles de la reacción. En general, las patentes de esa época proponen el uso de catalizadores que contienen metales del cuarto, sexto y octavo grupo de la tabla periódica mezclados con los metales del segundo y tercer grupo [15].
En la Fig. 2 se muestran los resultados más relevantes y se observa la relación entre el porcentaje de conversión de metano y la selectividad a metanol, las dos unidades expresadas en porcentaje en moles.

En la Fig. 2 se pueden identificar valores inferiores al 20,0\% de conversión de metano y como valor máximo de selectividad a metanol $55,0 \%$ los dos valores expresados en mol. En el caso de la referencia [16] no se reportaron valores de selectividad ni conversión; sin embargo, se indica el uso de óxidos metálicos de zinc, plomo y cromo como catalizadores en un rango de temperatura entre 250 y $500{ }^{\circ} \mathrm{C}$, a una presión entre 60 y 300 atmósferas.

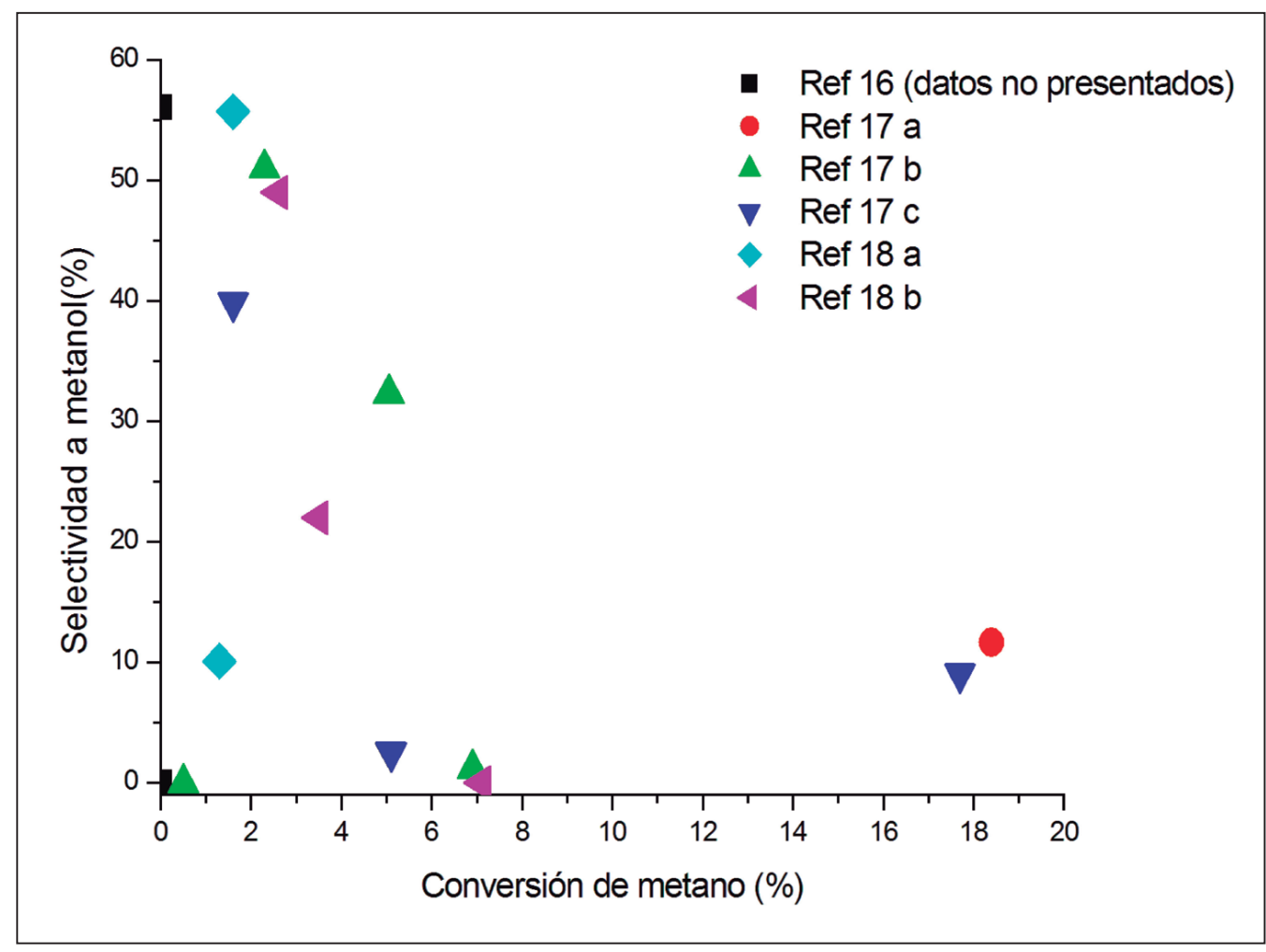

Fig. 2. Datos experimentales para la conversión de metano a metanol

Fuente: elaboración propia, a partir de [15]-[17]. 
En [15] se presenta en promedio el valor más alto de conversión de metano: $18,40 \%$ en mol con una selectividad de metanol de 11,65\% en mol. Las condiciones de trabajo empleadas fueron una mezcla en moles de oxígeno, metano y nitrógeno en una proporción de $13,0 \%, 35,0 \%$ y $52,0 \%$, respectivamente, utilizando catalizadores de cobre electrolítico a $350^{\circ} \mathrm{C}$ y 185 atm de presión. Además, modificando la alimentación del reactor a 3,7\%, $75,0 \%$ y $20,0 \%$ de oxígeno, metano y nitrógeno, respectivamente, y usando el mismo catalizador, se obtuvo el $5,0 \%$ de conversión de metano y una selectividad a metanol de $32,3 \%$ expresados en moles [15] . Lo anterior demuestra la importancia de los porcentajes de alimentación del reactor en el proceso de oxidación selectiva de metano a metanol, ya que bajo las mismas condiciones de temperatura, presión y catalizador, pueden ser obtenidos como productos de oxidación dióxido de carbono o monóxido de carbono, y solo varía este parámetro. Esto es debido a las reacciones continuas que se producen al generar la oxidación completa del metano y disminuir el rendimiento a productos útiles como el metanol, lo cual puede cambiarse y dependerá fundamentalmente del catalizador, la concentración de oxígeno y la temperatura [18]. Otro aspecto importante es la velocidad espacial por hora, ya que garantizando un proceso rápido en la superficie del catalizador, puede esperarse una mayor selectividad a productos intermedios de oxidación, como el metanol.

En [17] hay dos casos similares a los mencionados anteriormente. El primero, utilizando cobre como catalizador, muestra que con una alimentación al reactor de $2,8 \%, 82,0 \%$ y $15,0 \%$ de oxígeno, metano y nitrógeno, respectivamente, a $475{ }^{\circ} \mathrm{C}$ y $141 \mathrm{~atm}$ de presión se obtiene el $1,6 \%$ de conversión de metano en mol y una selectividad de $55,7 \%$ en mol, el valor más alto reportado. En el segundo proceso se modifican las condiciones de la alimentación a $8,6 \%, 55,0 \%$ y $26,0 \%$, de oxígeno, metano y nitrógeno, respectivamente, a $184 \mathrm{~atm}$ y se obtiene una conversión de metano de $3,5 \%$ (la más alta), pero con una selectividad a metanol de $22,0 \%$ (valor inferior), de la misma manera expresados en mol. Con base en esto, el tipo de reactor, temperatura, concentración de alimentación de oxígeno y flujo de gas afectan la eficiencia del proceso de oxidación selectiva de metano a metanol [17].

Teniendo en cuenta lo anterior, el uso de metales y óxidos metálicos como catalizadores han tenido y tienen gran interés debido a sus propiedades redox, las cuales a su vez están relacionadas con las propiedades ácido-base de los materiales catalíticos. Particularmente, en las reacciones de oxidación de hidrocarburos (selectivas o no) los metales u óxidos metálicos juegan un papel importante durante el proceso, ya que generan el paso fundamental de abstracción de hidrógeno para iniciar la oxidación; las condiciones del proceso influirán en los productos de la reacción, así como las relaciones de oxidante e hidrocarburo.

Según lo anterior, se evidencia el gran interés por lograr la oxidación selectiva de metano a metanol desde principios del siglo XX. Hoy en día es el mismo interés o aún mayor, en la medida en que [19] sugiere que el proceso es el Santo Grial en los procesos catalíticos. 
El uso de diferentes metales y óxidos de metales continuó a medida que avanzaba el siglo $\mathrm{XX}$; por ejemplo, el óxido de plata $\left(\mathrm{Ag}_{2} \mathrm{O}\right)$ y óxido de cromo $\left(\mathrm{Cr}_{2} \mathrm{O}_{3}\right)$ depositado sobre la piedra pómez fueron sugeridos como catalizadores para la oxidación de metano a metanol y formaldehído; el método propuesto involucra el paso de una mezcla de metano y oxígeno (98,2\% en volumen) sobre el catalizador a presiones entre 25 y 75 atm y temperaturas de entre 375 y $398^{\circ} \mathrm{C}$. Con el fin de aumentar la selectividad a formaldehído, en lugar de metanol, se empleó trióxido de molibdeno [20].

En otros estudios se evaluó la eficacia de una serie de catalizadores para la oxidación parcial de metano; dentro de los catalizadores incluidos están: $\mathrm{ZnO}, \mathrm{Co}_{3} \mathrm{O}_{4}, \mathrm{Ag}_{2} \mathrm{O}, \mathrm{MoO}_{3^{\prime}}$ $\mathrm{ThO}_{2}, \mathrm{Cr}_{2} \mathrm{O}_{3}, \mathrm{Ag} 2 \mathrm{O} / \mathrm{Cr}_{2} \mathrm{O}_{3}, \mathrm{CuO}, \mathrm{Al}_{2} \mathrm{O}_{3}, \mathrm{Fe}_{2} \mathrm{O}_{3}$, $\mathrm{MnO}_{2}, \mathrm{~V}_{2} \mathrm{U}_{5}, \mathrm{BaO}, \mathrm{U}_{2} \mathrm{O}_{8}, \mathrm{Al}_{3} \mathrm{PO}_{4}$, lana de vidrio, asbesto y piedra pómez. Por otra parte, los tubos del reactor eran construidos de níquel, cobre o acero inoxidable. Se realizaron evaluaciones de los catalizadores en el rango de temperatura de 375 a $500{ }^{\circ} \mathrm{C}$, las velocidades volumétricas fueron entre 15.000 y $50.000 \mathrm{~h}^{-1}$ y la carga de metal en el catalizador fue entre 0,5 y $50 \%$ en masa. Como era de esperarse, diferentes condiciones óptimas para la producción de metanol se encontraron para cada catalizador; sin embargo, los resultados más relevantes fueron una alta conversión de metano, 32,6\% en mol empleando $\mathrm{Cr} 2 \mathrm{O} 3$, mientras que $\mathrm{Ag}_{2} \mathrm{O}$ y $\mathrm{MoO}_{3}$ fueron más favorables para la producción de formaldehído [21]-[22].

En otros estudios con $\mathrm{Cr}_{2} \mathrm{O}_{3}$ en una relación de $3,0 \%$ y $10,0 \%$ en masa depositado en piedra pómez y $\mathrm{MoO}_{3}$, se encontró que al depositar el 3,0 \% del óxido en piedra pómez hubo una disminución en el rendimiento a formaldehído y metanol cuando se aumentó el contenido de oxígeno en la mezcla de gases dentro del rango de $2,5 \%$ a $5,0 \%$ a 200 atm. El valor más relevante obtenido fue una conversión de 48,2\% en mol de metano utilizando $\mathrm{Cr}_{2} \mathrm{O}_{3}$ [21]. Un hecho importante por resaltar es que en un estudio hecho para la oxidación del metano con oxígeno se encontró que la conversión total de metano en productos que contienen oxígeno no excede el 2,0 \% en mol [23]. Lo anterior se explica con base en que los otros productos de reacción son termodinámicamente más estables que los productos de oxidación intermedia.

En otros estudios se propuso una modificación de los procesos anteriores de oxidación parcial catalítica de metano con oxígeno sobre catalizadores heterogéneos, ahora en presencia de hidrógeno. En uno de los artículos divulgados el $\mathrm{CH}_{4}, \mathrm{O}_{2} \mathrm{y} \mathrm{H}_{2}$, se hicieron pasar simultáneamente por un catalizador de cromo y zinc entre 250 y 300 atm, y 350 y $370{ }^{\circ} \mathrm{C}$. Una típica composición de alimentación del reactor fue entre 4,0\% y 5,0\% de hidrocarburo, $95,0 \%$ y $96.0 \%$ de $\mathrm{H}_{2}$, y 0,3 y $0,5 \%$ de 02 [24]. Desafortunadamente, los datos de conversión de metano y selectividad a metanol no fueron reportados.

En los años 60 y 70, algunos investigadores sugirieron que aunque la oxidación de metano a metanol es factible, la cinética del proceso indica que la reacción homogénea en fase gaseosa favorece la formación de formaldehído en lugar de metanol. También encontraron que la formación de metanol se favoreció cuando fue catalizada por $\mathrm{Cr}_{2} \mathrm{O}_{3}$ sobre piedra pómez [25]. Los resulta- 
dos obtenidos a $430{ }^{\circ} \mathrm{C}$ y 1,5 atm revelaron que el $12,5 \%$ del metano que reacciona se convierte en metanol. En estos ensayos, se utilizó aire como fuente de oxígeno, manteniendo una relación entre metano y oxígeno de 10:1. Una conversión significativa de $\mathrm{CH}_{4}$ se presentó con perlas de vidrio, 10,3\% en mol, aunque también se registró que la selectividad era principalmente a óxidos de carbono. Se destaca que la presencia del catalizador, aunque no afecta la oxidación del metano, aumentó sustancialmente la selectividad a metanol [25].

De la misma manera, se encontró que el cloro gaseoso es un promotor efectivo de la oxidación parcial de metano. En un ensayo a $430{ }^{\circ} \mathrm{C}$, la oxidación de metano aumentó de $8,9 \%$ en mol en ausencia de cloro a $18,3 \%$ en mol con la presencia de este, en una proporción de alimentación de 6,6\% $\mathrm{v} / \mathrm{v}$; mientras que la cantidad de metano que reaccionó y se convirtió a metanol aumentó de $12,7 \%$ a 40,2 \% en mol. En [26] se sugiere que el cloro promovió la actividad catalítica del catalizador de $\mathrm{Cr}_{2} \mathrm{O}_{3}$ soportado en piedra pómez [26].

Por otra parte, en un estudio en el cual fue utilizado un catalizador de Pd soportado en $\mathrm{Al}_{2} \mathrm{O}_{3}$, se pudieron correlacionar los datos experimentales con una ecuación que asumió que el paso crítico de la reacción era la adsorción de metano y metanol en la superficie [27]. Este estudio es una muy buena aproximación al mecanismo Langmuir-Hinshelwood, donde la velocidad de la reacción es controlada por las especies adsorbidas en la superficie, lo cual indica claramente la necesidad de que los dos reactivos, el metano y el oxígeno, se adsorban sobre la super- ficie del material catalítico para su posterior reacción y formación de metanol.

Otras propuestas sugieren la adición de iniciadores homogéneos en la alimentación del reactor, como dimetil éter en proporciones entre $0,2 \%$ y $0,5 \% \mathrm{v} / \mathrm{v}$. El uso de esta sustancia disminuyó la temperatura de reacción en más de $100^{\circ} \mathrm{C}$ y duplicó la producción de productos de oxidación parcial. El catalizador utilizado fue un aluminosilicato modificado con óxidos metálicos y fosfatos. Averbuch et al. sostienen que el dimetil éter actuó como un iniciador y produjo radicales metilo y óxidos [28]-[29].

La ICI, que fue la principal licenciadora de metanol a partir de la tecnología de gas de síntesis, también tiene una patente para la oxidación parcial de metano a metanol y formaldehído. Una mezcla de metano (96,0\%-98,0 \% v/v) y oxígeno puro se pasan por diferentes catalizadores, incluyendo óxido de molibdeno a $50 \mathrm{~atm}$ y temperaturas en el rango de $430-500{ }^{\circ} \mathrm{C}$. Los datos obtenidos muestran que, a pesar de que es posible producir metanol y formaldehído, las conversiones y selectividades obtenidas son muy bajas [29].

De la misma manera, en 1975, la industria British Gas Corporation llevó a cabo una patente para la invención de un catalizador que permitiera la oxidación parcial de metano a metanol y formaldehído [30]. El catalizador para la oxidación estaba compuesto de óxido de tungsteno o molibdeno, junto con el óxido de un metal de valencia variable, como cobre, hierro, níquel, cobalto, cromo, vanadio o bismuto. En este caso, la evaluación de los materiales catalíticos se llevó a cabo con una mezcla 
de metano y oxígeno, los cuales eran pasados sobre el catalizador de oxidación a una presión de 5 atm o más y en el rango de temperatura de $460-550{ }^{\circ} \mathrm{C}$. Las condiciones del proceso eran seleccionadas de modo tal que el oxígeno no superara el $75,0 \%$ de la alimentación en el reactor. Una cantidad significativa del producto oxigenado consistió de compuestos útiles, como el metanol, formaldehído, acetaldehído y etileno [30].

En la Fig. 3 se resumen los resultados más relevantes de conversión de metano versus la selectividad a metanol; las dos unidades expresadas en porcentaje en moles. Es importante mencionar que dos de las referencias citadas no exhiben valores de selecti- vidad a metanol; sin embargo se presentan, porque sí reportan valores de conversión de metano empleando óxidos metálicos como el cromo, zinc y molibdeno.

En la Fig. 3 la tendencia está dada por valores inferiores al $10,0 \%$ en mol de conversión de metano y valores de selectividad a metanol muy variantes, lo cual indica que las investigaciones realizadas no alcanzaron altos rendimientos. Se destaca como mayor valor de conversión de metano 48,2\% en mol y como mayor valor de selectividad $75,0 \%$; no obstante, el mayor valor de rendimiento (producto entre conversión y selectividad) de todos los ensayos realizados fue del $2,62 \%$, lo cual muestra la gran dificultad de controlar el proceso.

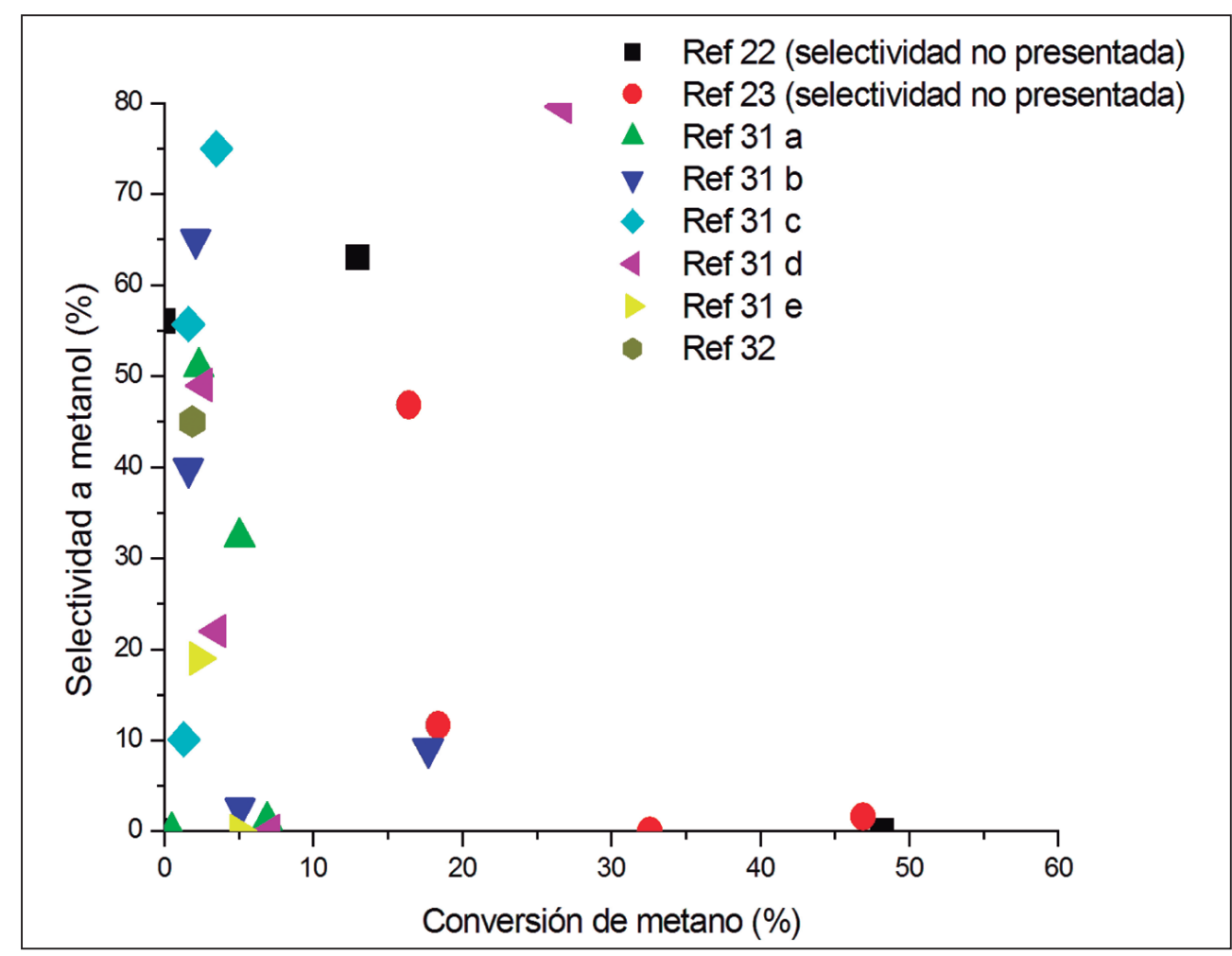

Fig. 3. Datos experimentales para la conversión de metano a metanol Fuente: elaboración propia, a partir de [21]-[22],[30]-[31]. 
Como se mencionó anteriormente, [21] y [22] no presentan valores de selectividad a metanol, mientras que [30] muestra cinco resultados diferentes. Dentro de los resultados se destaca que el óxido de molibdeno se usa como soporte que cumple la función de incrementar el área superficial de los materiales catalíticos y a su vez es el vehículo de la fase activa, que en este caso fue $\mathrm{ZnO}, \mathrm{Fe}_{2} \mathrm{O}_{3}$, el $\mathrm{UO}_{2}, \mathrm{VO}_{2}$. Las comparaciones de los resultados son un poco difíciles, ya que así como cambian las especies activas, también lo hacen las condiciones del proceso; además, como se dijo, estas influyen directamente en la actividad de los materiales catalíticos y, por ende, en los resultados obtenidos.

Por citar un ejemplo, la mayor selectividad a metanol se logró con el catalizador $\mathrm{MoO}_{3}-\mathrm{UO}_{2}$, con un valor de $75,0 \%$ y una conversión de metano de $3,5 \%$, los dos valores en moles (ref. $31 \mathrm{c}$ ), con una alimentación del reactor de $3,9 \%$ y $96,1 \%$ v/v de $\mathrm{O}_{2}$ y $\mathrm{CH}_{4}$, respectivamente, a $470{ }^{\circ} \mathrm{C}$ y $54 \mathrm{~atm}$ de presión con una velocidad espacial horaria de $23.200 \mathrm{~h}^{-1}$. Por otra parte, los resultados obtenidos por el catalizador $\mathrm{MoO}_{3}-\mathrm{VO}_{2}$ fueron una selectividad a metanol de $49,0 \%$ en mol, con una conversión de metano de $2,6 \%$ en mol (ref. 31 d), con una alimentación del reactor de $2,4 \% \mathrm{v} / \mathrm{v}$ de $\mathrm{O}_{2}$ y $97,6 \%$ $\mathrm{v} / \mathrm{v}$ de $\mathrm{CH}_{4}$ a $493^{\circ} \mathrm{C}$ y $51 \mathrm{~atm}$ y una velocidad espacial horaria de $47.600 \mathrm{~h}^{-1}$.

Con base en los resultados, es evidente que el óxido de vanadio soportado en óxido de molibdeno produce un rendimiento menor en relación con el óxido de uranio; sin embargo, todas las condiciones del proceso son diferentes, lo cual motiva la siguiente pregunta: ¿cuál de los dos catalizadores es mejor? Partiendo de los resultados, no es posible dar una respuesta definitiva, para esto sería necesario comparar los dos catalizadores bajo las mismas condiciones.

Por otra parte, se destaca que solo el óxido de molibdeno $\mathrm{MoO}_{3}$ sin especies activas dio el menor valor de selectividad a metanol, $19,0 \%$ en mol, con una conversión de metano de $2,3 \%$ en mol (ref. 31 e) con una alimentación del reactor de $2,0 \% \mathrm{v} / \mathrm{v}$ de $\mathrm{O}_{2}$ y el $98,0 \% \mathrm{v} / \mathrm{v}$ de $\mathrm{CH}_{4}$ a $460{ }^{\circ} \mathrm{C}$ y $54 \mathrm{~atm}$ con una velocidad espacial horaria de $25.500 \mathrm{~h}^{1}$.

\subsection{Metales y óxidos metálicos soportados como catalizadores}

En este apartado se presentan resultados de investigaciones hechas para lograr la oxidación selectiva de metano a metanol, en las cuales el interés se centró principalmente en el uso de materiales catalíticos soportados. En estos materiales se diferencian dos componentes principales: el soporte y la fase activa. Algunos autores sugieren que el soporte solamente es el vehículo sobre el cual se ubica la fase activa, mientras que otros manifiestan que pueden hacer parte del proceso catalítico. Según esto, se presentan y discuten los resultados conseguidos para la obtención de metanol a partir de la oxidación directa de metano.

Leonov et al. [31] evaluaron un catalizador de $\mathrm{Zn}-\mathrm{Ni}-\mathrm{Cd}$ a $350^{\circ} \mathrm{C}$ y $100 \mathrm{~atm}$. Para esto se empleó una mezcla de metano y oxigeno que contenía el $3,0 \% \mathrm{v} / \mathrm{v}$ del oxidante; el producto de reacción generó entre un $54,0 \%$ y un $58,0 \%$ en mol de metanol; no obstante, no se muestra el valor de conversión de metano. En otro estudio, Liu et al. [32] evaluaron un catalizador de $\mathrm{MoO}_{3}-\mathrm{SiO}_{2}$. Dentro de los 
resultados relevantes, se encontró que a $600^{\circ} \mathrm{C}$ y $1 \mathrm{~atm}$, la selectividad a metanol fue del $46,8 \%$ en mol, con una conversión de $\mathrm{CH}_{4}$ de $16.4 \%$ en mol. De la misma manera, Iwamoto [33] evaluó mezclas gaseosas que contenían $\mathrm{CH} 4, \mathrm{~N} 2 \mathrm{O}$, vapor de agua y helio en una proporción molar de 1.0/2.0/4.7/2.3 a través de un catalizador de V205$\mathrm{SiO} 2$ a 450,500 y $550{ }^{\circ} \mathrm{C}$. Los valores de selectividad a metanol fueron de $0,0 \%$, $39,8 \%$ y $85,6 \%$ en mol, con conversiones de metano de $0,5 \%, 1,6 \%$ y $11,2 \%$ en $\mathrm{mol}$, respectivamente.

Por otra parte, con el catalizador $\mathrm{MoO}_{3}$ $\mathrm{SiO}_{2}$ y con una mezcla similar de gases: $\mathrm{CH}_{4}, \mathrm{~N}_{2} \mathrm{O}$, vapor de agua y helio en relaciones 1.0/2.0/1.67/5.33 a $550{ }^{\circ} \mathrm{C}$ y $1 \mathrm{~atm}$, se generó una selectividad a metanol de 79,6\% en mol con una conversión total de $\mathrm{CH}_{4}$ de $26,7 \%$ en mol [34].

En otra investigación realizada para evaluar la obtención de metanol, Gesser et al. [35] emplearon gas natural y metano puro, con $5,0 \% \mathrm{v} / \mathrm{v}$ de oxígeno en la mezcla de reacción a $450{ }^{\circ} \mathrm{C}$ y $50 \mathrm{~atm}$; se obtuvo una selectividad a metanol de $85,0 \%$ en mol, con una conversión de gas natural de $10,0 \%$ en mol y $80,0 \%$ de selectividad a metanol cuando se empleo metano puro, con niveles de conversión similar.

De acuerdo con esto, sobre el papel tan importante que tienen las condiciones del proceso, en 1990 Hunter et al. [36] presentaron el efecto de la composición de la pared del reactor en la oxidación homogénea del gas natural evaluada a una presión de 30 atm y una temperatura entre 350 y $400{ }^{\circ} \mathrm{C}$; de la misma manera, se evaluó el efecto de catalizadores sólidos en la oxidación del gas natural en un sistema heterogéneo a 30 atm y diferentes temperaturas.

La alimentación del reactor consistió en una mezcla binaria de gas natural o metano y oxígeno; en algunos ensayos una pequeña cantidad de nitrógeno fue agregado al metano o gas natural para que actuara como un estándar interno que permite una estimación más precisa de la conversión [36]. Sorprendentemente, los valores de selectividad a metanol fueron superiores al $89,5 \%$ en mol, cuando el reactor empleado era construido con vidrio, con una conversión de metano del $10,0 \%$ en mol a $50 \mathrm{~atm}$ de presión y $350^{\circ} \mathrm{C}$. Cuando se empleó teflón se obtuvo el 8,0\% en mol de conversión de metano y el $93,0 \%$ en mol de selectividad a metanol, a $30 \mathrm{~atm}$ y $361^{\circ} \mathrm{C}$. Además, cuando se usaron condiciones similares, pero con otros materiales como acero inoxidable, se obtuvo el $94,5 \%$ en mol de selectividad a metanol y $2,0 \%$ en mol de conversión de metano; mientras que al utilizar plata y cobre, se consigupo el $20,0 \%$ $y$ el $22,0 \%$, respectivamente, en mol de selectividad a metanol y $1,5 \%$ y $1,0 \%$ en mol de conversión de metano [36]. De acuerdo con los resultados, se puede mostrar que el tipo de material del reactor afecta tanto la selectividad a metanol como la conversión de metano, debido a los diferentes calores de adsorción de cada molécula sobre cada tipo de material, lo cual puede direccionar el proceso a uno u otro producto.

De la misma manera, catalizadores de $\mathrm{Cu} /$ $\mathrm{SiO}_{2}, \mathrm{Co} / \mathrm{Al}_{2} \mathrm{O}_{3}, \mathrm{TiO}_{2}$ y $\mathrm{SnO}_{2}$ fueron evaluados a temperaturas entre 250 y $400{ }^{\circ} \mathrm{C}$. Los resultados indican que el $\mathrm{Cu} / \mathrm{SiO}_{2}$ logró una selectividad a metanol de $95,0 \%$ en mol con 
una conversión de metano de 6,0 \% en mol, similar a la que se obtiene con $\mathrm{Co} / \mathrm{Al}_{2} \mathrm{O}_{3^{\prime}}$ $96,0 \%$ y $6,0 \%$ en mol, respectivamente; mientras que el catalizador $\mathrm{TiO} 2$ no produce metanol y logra una conversión de $2,5 \%$ en mol de metano. Finalmente, $\mathrm{SnO}_{2}$ alcanzó un valor de selectividad a metanol de $83,0 \%$ y una conversión de metano de $4,0 \%$, los dos porcentajes en moles [36].

Por otra parte, Spencer et al. [38] evaluaron el efecto del sodio en la oxidación parcial de metano sobre $\mathrm{MoO}_{3}-\mathrm{SiO}_{2}$ en presencia de oxígeno molecular, así como la propuesta de un análisis cinético en el cual el metano se oxida directamente a dióxido de carbono y formaldehído. El estudio presentó un mecanismo de reacción para la conversión de metano, en el cual el ataque inicial sobre el gas es una reacción con los radicales Mo-O* generados térmicamente, lo que produce la extracción de un hidrógeno y eso genera un intermedio metoxi [37]. En este paso es posible continuar hacia metanol, formaldehído, o completar la oxidación a dióxido de carbono.

Lyon et al. [38] describieron el uso de un catalizador a base de sodalita de hierro para la oxidación de metano a metanol, utilizando aire a altas presiones y temperaturas entre 380 y $480{ }^{\circ} \mathrm{C}$. Dentro de los resultados se destaca una selectividad de $70,0 \%$ en mol a metanol con una conversión de metano de $5,7 \%$ en mol a 416 ${ }^{\circ} \mathrm{C}$ y 55 bares con una relación de metano oxígeno de 3:1. De la misma mane$\mathrm{ra}$, Sen et al. [39] sugirieron una idea muy aceptada: el metano es quimisorbido en un puente siloxano y se disocia generando dos posibles intermediarios; uno de estos se descompone para generar formaldehído, mientras que el otro sugiere que los radicales metilo en fase gaseosa forman etano. En este documento no se presenta selectividad a metanol.

Más adelante, Walker et al. [40] presentaron resultados de la oxidación directa de metano a metanol, utilizando como catalizadores $\mathrm{Fe}$ /sodalita, alúmina, $\mathrm{Pd} / \mathrm{MgO}, \mathrm{MoO}_{3} /$ $\mathrm{UO}_{2}$ y $\mathrm{SnO}_{2}$ a temperaturas entre 380 y 550 ${ }^{\circ} \mathrm{C}$. La alimentación del reactor tubular de alta presión fue de $23,8 \% \mathrm{v} / \mathrm{v}$ de aire y $76,2 \%$ $\mathrm{v} / \mathrm{v}$ de metano [40]. Se destaca que la mayor conversión de metano fue obtenida por el catalizador $\mathrm{MoO}_{3} / \mathrm{UO}_{2}, 5,0 \%$ en mol con una selectividad a metanol de $38,0 \%$ en mol

Es importante mencionar que Hall et al. [41] mostraron que es evidente que los óxidos y los catalizadores de óxidos funcionan bajo condiciones que están dominadas por la química de radicales y las reacciones en fase gaseosa. Estos dos tipos de procesos imponen graves limitaciones en las selectividades alcanzables para el metanol a partir de la oxidación directa de metano.

Por otro lado, Raja et al. [42] presentaron los resultados de la conversión de metano a metanol y formaldehído empleando una mezcla de 02/hidroperóxido de terc-butilo como oxidantes (TBHP), en condiciones ambientales con ftalocianinas de hierro, cobre y cobalto encapsuladas en zeolitas $X$ y Y. El proceso se llevó a cabo a $546^{\circ} \mathrm{C}$ durante 12 horas y se se alimentó el reactor con metano a una presión de 50 psi y aire a una presión de 100 psi; la relación entre el catalizador y el oxidante fue de 0,5 y 0,75 gramos, respectivamente. Dentro de los resultados se destaca que la zeolita $X$ con hierro 
alcanzó un porcentaje de conversión de metano de $4,9 \%$ en mol y una selectividad a metanol de $52,6 \%$ en mol. La misma zeolita, pero con cobre, permitió obtener el $4,2 \%$ y $51,5 \%$ en mol de conversión de metano y selectividad a metanol, respectivamente. Adicionalmente, la zeolita $Y$ con cobre alcanzó $3,9 \%$ en mol de conversión de metano y $53,5 \%$ en mol de selectividad hacia el alcohol. Estos autores sugieren que el catalizador sólido es esencial para la reacción de oxidación, ya que los ensayos llevados a cabo en ausencia de catalizador mostraron una conversión de metano inferior al 0,1\% en mol bajo condiciones idénticas; además, ftalocianinas de hierro y cobre, donde todos - la mayoría de hidrógenos que han sido sustituidos poseen una alta actividad catalítica en la oxidación de metano utilizando fuentes de $\mathrm{O}_{2} /$ TBHP como oxidantes [42].
Por su parte, Taylor et al. publicaron un documento acerca del enfoque en el diseño de catalizadores para la oxidación parcial de metano. Tal enfoque consiste en identificar los componentes de catalizadores de metales u óxidos de metales que activan el metano y el oxígeno, pero no destruyen fácilmente el metanol, que es el producto deseado. En efecto, una serie de catalizadores a base de $\mathrm{MoO}_{3}$ y $\mathrm{WO}_{3}$ fueron preparados por impregnación con soluciones que contienen los iones metálicos [43].

Los resultados más relevantes, cuando el reactor se alimentó en una proporción de 23.0/3.0/5.0 \%v/v de metano, oxígeno y helio, respectivamente, a una presión de 15 bar y una velocidad espacial horaria de 5.000 $\mathrm{h}^{-1}$ se presentan en la Tabla 2.

Tabla 2. Oxidación del metano a metanol

\begin{tabular}{|c|c|c|c|}
\hline Catalizador & $\begin{array}{c}\text { Temperatura } \\
\left({ }^{\circ} \mathrm{C}\right)\end{array}$ & $\begin{array}{l}\text { Conversión de } \\
\mathrm{CH}_{4}(\% \text { en mol })\end{array}$ & $\begin{array}{c}\text { Selectividad a } \\
\mathrm{CH}_{3} \mathrm{OH} \text { (\%en mol) }\end{array}$ \\
\hline $\mathrm{Cu} / \mathrm{MoO}_{3}$ & 450 & 0,6 & 19,0 \\
\hline $\mathrm{Fe} / \mathrm{MoO}_{3}$ & 450 & 0,5 & 14,0 \\
\hline $\mathrm{V} / \mathrm{WO}_{3}$ & 450 & 0,8 & 16,0 \\
\hline $\mathrm{Ga}_{2} \mathrm{O}_{3} / \mathrm{MoO}_{3}$ & 455 & 3,0 & 22,0 \\
\hline $\begin{array}{l}\text { Tubo vacío } \\
\text { Lecho de cuarzo }\end{array}$ & 450 & 8,1 & 29,0 \\
\hline
\end{tabular}

Fuente: elaboración propia, a partir de [43].

En el reactor sin catalizador, tanto la conversión de metano y la selectividad a metanol son mayores con respecto a los demás procesos que evaluaban catalizadores. Esta tendencia de conversión puede estar relacionada con el tiempo de permanencia dentro de la zona climatizada del reactor, que es menor para la lana de cuarzo. En general, se esperaría que la selectividad a metanol incrementara a mayor conversión de metano; sin embargo, la presión parcial del oxígeno y el aumento de la superficie calentada dentro 
del lecho de cuarzo y el material catalítico pueden favorecer la combustión de metanol, y reducir así la selectividad [43].

De acuerdo con lo anterior, se evidencia la gran cantidad de trabajos y la inclusión de diferentes tipos de materiales catalíticos, así como la variación de las condiciones de reacción en busca de altos valores de conversión y selectividad a metanol. En la Fig. 4 se presentan los resultados de conversión de metano y selectividad a metanol expresados en porcentaje en moles.

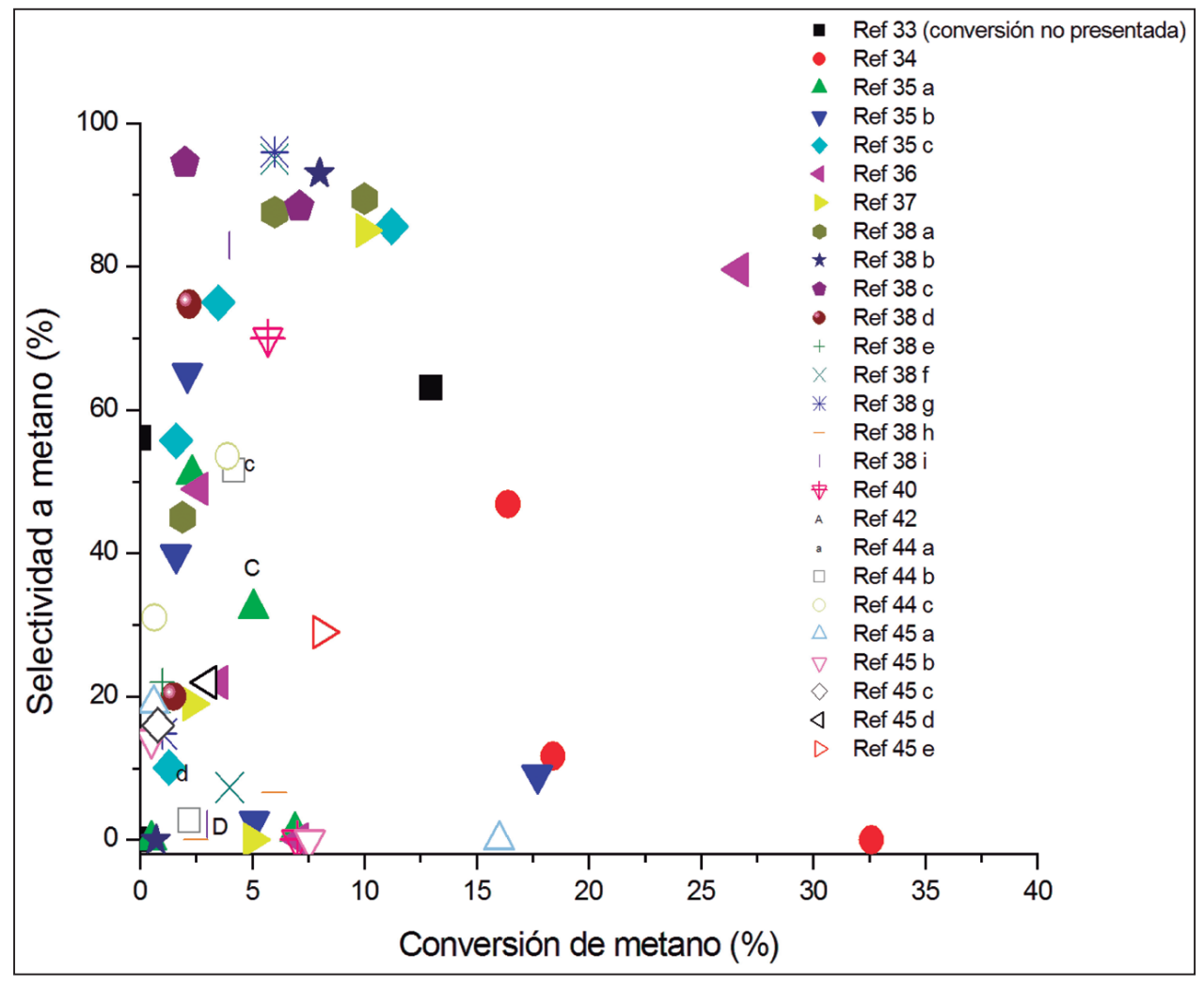

Fig. 4. Datos experimentales para la conversión de metano a metanol Fuente: elaboración propia, a partir de [32]-[37],[39],[41],[43]-[44].

Como puede verse en la Fig. 4, la tendencia está dada por valores inferiores al $30,0 \%$ en mol de conversión de metano y valores de selectividad a metanol entre el $10,0 \%$ y el $95,0 \%$ en mol. En el caso de la selectividad, hay valores altos pero a bajas conversiones de metano, lo cual influye en el rendimiento del proceso. Se destaca como mayor valor de conversión de metano un $26,7 \%$ en mol y como mayor valor de selectividad un $96,0 \%$ en mol; no obstante, el mayor valor de rendimiento (producto entre conversión y selectividad) de todos los ensayos realizados fue el $21,25 \%$, lo cual muestra la gran dificultad de controlar proceso.

Asimismo, se puede ver en la Fig. 4 que el número de estudios reportados empleando 
materiales catalíticos soportados fue mayor que cuando se usaban metales u óxidos metálicos. Es importante resaltar que, a pesar de los intentos por lograr grandes conversiones de metano y un alto nivel de selectividad a metanol, esto no fue posible ya que la tendencia del gráfico muestra una alta selectividad hacia el alcohol cuando las conversiones de metano son bajas. Esto es debido a las reacciones competitivas y secuenciales que se producen durante la oxidación selectiva de metano a metanol, las cuales favorecen otros productos de oxidación, como el dióxido de carbono o monóxido de carbono. También se destaca la variedad de procedimientos implementados, que van desde el cambio de las variables de proceso: temperatura, presión, mezcla del alimento del reactor o evaluar el tipo de material utilizado en el reactor, como acero inoxidable, cuarzo, teflón, entre otros, los cuales influencian y reacciona con los reactivos o productos del proceso, aspecto que se refleja en el rendimiento de la reacción catalizada.

\subsection{Zeolitas y otros materiales catalíticos}

Continuando con la intención de conocer los avances del proceso de oxidación selectiva de metano a metanol, en un estudio publicado en 2002, se presentaron resultados de la oxidación de metano a temperaturas entre $575^{\circ} \mathrm{C}$ y $625^{\circ} \mathrm{C}$, empleando como catalizador sílice precipitada y diferentes presiones parciales de oxígeno [44]. En dicho trabajo se menciona que en este sistema, un alto nivel de selectividad a los productos deseados, (metanol y formaldehído) solo se logra a baja conversión de metano, ya que reacciones secuenciales generan la disminución de la selectividad. El efecto del aumento de la conversión de metano puede ser explicado en términos de la estabilidad relativa del gas (C-H fuerza de enlace 438 $\mathrm{kJ} / \mathrm{mol}$ ) y formaldehído (C-H fuerza de enlace $364 \mathrm{~kJ} / \mathrm{mol}$ ) [44].

Esto tiene un gran sentido, en la medida en que se entiende que es necesaria más energía para la activación de metano, mientras que una vez formado el metanol o el formaldehido pueden ser más fácilmente oxidados. Además, será necesario considerar las propiedades del material catalítico como porosidad, ya que si la reacción de oxidación selectiva de metano se lleva a cabo dentro de los poros de los catalizadores y no en la superficie, es muy probable que durante el proceso de desorción y difusión de los productos desde el interior de los poros a la superficie exterior y luego a la fase fluida, quizá estos productos se pueden oxidar en otros, como el dióxido de carbono, y reducir así la selectividad a metanol o formaldehído. En los ensayos, la conversión varió dependiendo de la velocidad espacial horaria de los reactivos, la cual se mantuvo en un rango entre 10.000 y $60.000 \mathrm{~h}^{-1}$. Cabe mencionar que el metanol no fue observado como producto de la reacción [44].

En otro estudio realizado, Zhang et al. [45] evaluaron el diseño de un reactor para la oxidación del metano a metanol. La oxidación en fase gaseosa de metano se realizó a temperaturas entre 380 -y $500{ }^{\circ} \mathrm{C}$ y a presiones entre 1,0 y $5,0 \mathrm{MPa}$ en un reactor de flujo de alta presión que contenía una línea de cuarzo de $460 \mathrm{~mm}$ de longitud con un diámetro interno 
de $6,7 \mathrm{~mm}$, que terminaba en una tubería de acero inoxidable. Se empleó una unión con tuerca de bloqueo que contenía un orín de viton presionado para evitar la fuga de gas en el espacio anillado [45]. Los productos de la oxidación en fase gaseosa de metano fueron principalmente $\mathrm{CH}_{3} \mathrm{OH}, \mathrm{CO}, \mathrm{CO}_{2}, \mathrm{C}_{2} \mathrm{H}_{6}$ y en ocasiones, $\mathrm{C}_{2} \mathrm{H}_{4}$. Se obtuvo valores de selectividad a metanol de $63,0 \%$ en mol, cuando la conversión de metano fue $13,0 \%$ también en mol [45]. Se propuso entonces que las razones más probables de los resultados obtenidos incluyen: 1) la forma de calentamiento, lo que traería una nueva variable para el perfil de temperatura del reactor; 2) el aislamiento del anillado de separación entre el interior y el exterior de la línea de cuarzo y el tubo de acero inoxidable; 3 ) la línea de cuarzo, y 4) el uso de un separador, que pudo afectar el patrón de flujo y acortar el tiempo de residencia.

Por otra parte, Chen et al. [46] evaluaron la oxidación parcial de metano a metanol mediante la descarga de una barrera dieléctrica a través de un catalizador de $\mathrm{Fe}_{2} \mathrm{O}_{3}$ $\mathrm{CuO} / \mathrm{Y}^{-} \mathrm{Al}_{2} \mathrm{O}_{3}$. El catalizador se combinó con plasma en dos configuraciones diferentes: primero plasma y luego el proceso de catálisis (IPC), o primero catálisis y luego plasma (PPC). Estos investigadores propusieron que la aplicación de plasma no térmico como una descarga de barrera dieléctrica (DBD) puede ser una importante alternativa para producir productos químicos bajo condiciones amigables ambientalmente. El efecto sinérgico del plasma y la catálisis (IPC) se pudo observar en ambas combinaciones. La combinación plasma-catálisis resultó ser más efectiva, y alcanzó una conversión de metano de $46,9 \%$ en mol y una selectividad a metanol de 1,6\% en mol, con la desventaja de que fue el catalizador menos estable de la configuración PPC [46].

Por otra parte, [47] estudiaron en 2011 el tratamiento alcalino de zeolitas (Na-ZSM-5) y encontraron que la superficie externa de la zeolita se incrementó con el aumento de la concentración de $\mathrm{NaOH}$ y el tiempo de pretratamiento; además, al cargar estos materiales con cobalto se halló una relación lineal entre el número de especies de óxido de cobalto y la superficie externa de la zeolita.

Los resultados mostraron una selectividad hacia el metanol entre el $50,0 \%$ y el $75,0 \%$ en mol, pero no presentaron porcentajes de conversión de metano. El comportamiento presentado indicó que la cantidad de metanol producida aumenta con la cantidad de óxidos de cobalto presentes en las muestras; por tal razón, se concluye que un aumento de la superficie por tratamiento alcalino facilita el alojamiento de una mayor cantidad de óxidos de cobalto sobre la superficie exterior, que a su vez aumenta la selectividad hacia el metanol [47]. Por otra parte, propusieron que la desorción de metanol podría ser facilitada por la mayor meso porosidad en las zeolitas [47].

De igual manera, en 2011 se estudió la estructura de catalizadores de pirofosfato de cobre y hierro preparados con diversas relaciones de metales y diferentes condiciones de pretratamiento; los materiales se evaluaron en la oxidación selectiva de metano a formaldehído y metanol empleando $\mathrm{O}_{2} \mathrm{O}_{2} \mathrm{O}$ como agentes oxidantes [48]. Los resultados muestran valores de selectividad a metanol de $1,2 \%, 2,5 \%$ y $10,1 \%$ en mol con conversiones de metano de $6,9 \%, 5,1 \%$ y $1,3 \%$ en mol, 
respectivamente [48]. En este sentido, hay tres variables que fueron estudiadas: 1) la proporción de cobre/hierro; 2) la temperatura de calcinación, y 3 ) la duración del periodo de calcinación a la misma temperatura. Los anteriores parámetros influyen en la estructura del material catalítico incluyendo la estructura cristalina, el tamaño de los poros y la formación de óxidos de los componentes activos del catalizador. De acuerdo con los resultados, el proceso de síntesis más favorable para obtener el mejor rendimiento fue a una temperatura de $750^{\circ} \mathrm{C}$ de calcinación durante cuatro horas, con una relación de hierro y cobre de 0:3.

En 2012, en [49] se evaluó la oxidación selectiva de metano a metanol empleando ácido acético como solvente, a una presión de 2,5 MPa de $\mathrm{CH}_{4}, 0,4 \mathrm{MPa} \mathrm{O}_{2}$ y 0,4 MPa $\mathrm{N}_{2}$ a $130^{\circ} \mathrm{C}$, durante tres horas y con una relación de volumen de $\mathrm{CH}_{3} \mathrm{COOH}$ y $\mathrm{H}_{2} \mathrm{O}$ de 1:4. Bajo estas condiciones, la producción de metanol alcanzó a ser 1650 umoles en un reactor de $250 \mathrm{~mL}$. Los mismos autores proponen que el disolvente juega un papel muy importante en la oxidación selectiva de metano a metanol en fase líquida, ya que ofrece un ambiente adecuado para la reacción y tiene características de bajo costo, benignidad ambiental y genera baja corrosión [49].

Por otra parte, el efecto de las estructuras de óxidos de vanadio sobre las propiedades fotocatalíticas para la oxidación parcial de metano a metanol fue estudiado; se prepararon tamices moleculares mesoporosos que contenían vanadio bajo condiciones ácidas y básicas, empleando el método de impregnación [50]. Se destaca que los sistemas fotocatalíticos ofrecen la posibilidad de promover muchas reacciones difíciles, incluso a temperatura ambiente, lo cual trae ventajas como bajo consumo de energía, estabilidad del catalizador y seguridad del reactor [50]. Los resultados más relevantes se encuentran en la Tabla 3, en la cual se puede ver que la mayor selectividad para el metanol fue $87,6 \%$ en mol con una conversión de metano del $6,0 \%$ en mol.

Tabla 3. Oxidación de metano a metanol

\begin{tabular}{|c|c|c|c|}
\hline Catalizador & Oxidante & $\begin{array}{c}\text { Conversión de } \mathrm{CH}_{4} \\
\text { (\%en mol) }\end{array}$ & $\begin{array}{c}\text { Selectividad } \\
\mathrm{CH}_{3} \mathrm{OH} \\
\text { (\%en mol) }\end{array}$ \\
\hline V-MCM-41(ácido) & $\mathrm{O}_{2}$ & 7.1 & -- \\
\hline V-MCM-41(base) & $\mathrm{O}_{2}$ & 5.0 & -- \\
\hline V-MCM-41(ácido) & NO & 6.0 & 87.6 \\
\hline V-MCM-41(base) & NO & 0.7 & -- \\
\hline Imp-V/MCM-41(ácido) & NO & 7.1 & 88.4 \\
\hline Imp-V/MCM-41(base) & NO & 2.2 & 74.7 \\
\hline
\end{tabular}

Fuente: elaboración propia, a partir de [50]. 
Se propone para la oxidación fotocatalítica que las especies tetraédricas de $\mathrm{VO}_{4}$ soportadas en la superficie de MCM-41 reaccionen con el $\mathrm{CH}^{4}$, lo que conduce a una ruptura del enlace $\mathrm{C}-\mathrm{H}$ y genera $\left(\mathrm{V}^{4+}-\mathrm{OH}\right)$ y el radical metilo $\left(\mathrm{CH}_{3} \cdot\right)$ [50]. Sobre la base de las propuestas de mecanismos, este estudio sugiere que un oxígeno del material catalítico se incorpora a la estructura del metano y el oxígeno entra de nuevo en el catalizador a partir del agente oxidante, lo cual es coherente con el mecanismo Marx-van Krevelen.

De la misma manera, la oxidación parcial de metano mediante oxígeno molecular y óxido nitroso fue estudiada en presencia de cantidades catalíticas de hetero-polioxometalátos de fórmula general $\left[\mathrm{PW}_{11} \mathrm{MO}_{39}\right]_{(7-n)}$ donde $\mathrm{M}=\mathrm{Co}(\mathrm{II}), \mathrm{Ni}(\mathrm{II})$ y $\mathrm{Fe}(\mathrm{III})$. La reacción de oxidación se realiza a presión atmosférica y a temperaturas entre $500^{\circ} \mathrm{C}$ y $650^{\circ} \mathrm{C}$. Los principales productos de reacción observados fueron metanol, formaldehído, óxidos de carbono y agua [51].

Los resultados más relevantes se encuentran en la Tabla 4, y se puede ver que la mayor selectividad para el metanol es 17,8\% en mol, con una conversión de metano de $0,80 \%$ en mol cuando la temperatura de reacción fue $600^{\circ} \mathrm{C}$ [51].

Tabla 4. Oxidación de metano a metanol

\begin{tabular}{|l|l|l|l|}
\hline \multirow{2}{*}{ Catalizador } & \multicolumn{1}{|c|}{ Temperatura } & \multicolumn{1}{|c|}{$\begin{array}{c}\text { Conversión de } \\
\mathbf{C H}_{\mathbf{4}}(\% \text { en } \mathbf{~ m o l})\end{array}$} & \multicolumn{1}{c|}{$\begin{array}{c}\text { Selectividad } \\
\mathbf{C H}_{\mathbf{3}} \mathbf{O H} \text { (\% en mol) }\end{array}$} \\
\hline \multirow{2}{*}{$\mathrm{CsPW}_{11} \mathrm{Fe}$} & 873 & 0,80 & 17,8 \\
\cline { 2 - 4 } & 923 & 4,0 & 7,3 \\
\hline \multirow{2}{*}{$\mathrm{CsPW}_{11} \mathrm{Co}$} & 873 & 1,0 & 14,8 \\
\cline { 2 - 4 } & 923 & 6,0 & 6,6 \\
\hline \multirow{2}{*}{$\mathrm{CsPW}_{11}$ IN } & 873 & 3,0 & 2,2 \\
\cline { 2 - 4 } & 923 & 7.0 & -- \\
\hline
\end{tabular}

Fuente: elaboración propia, a partir de [51].

Los resultados muestran que la naturaleza de los productos de la reacción de oxidación parcial de metano depende de la capacidad reductora del catalizador. En el catalizador $\left(\mathrm{PW}_{11} \mathrm{Ni}\right)$, el $\mathrm{CH}_{4}$ es totalmente oxidado a CO2. Sin embargo, en catalizadores con clústeres reducidos de hierro y cobalto, el $\mathrm{CH} 4$ se puede convertir de forma selectiva a $\mathrm{CH}_{3} \mathrm{OH}, \mathrm{HCHO}$ y $\mathrm{CO}$ con una pequeña cantidadde $\mathrm{CO}_{2}$ [52].
Por otra parte, se realizaron ensayos para la oxidación de metano, en los cuales una muestra de zeolita de $0,5 \mathrm{~cm}^{3}(0,36 \mathrm{~g}$.) fue colocado en un reactor de cuarzo con diámetro interno de $7,0 \mathrm{~mm}$. Antes de los ensayos, el catalizador fue pretratado con aire a $550^{\circ} \mathrm{C}$ durante 30 minutos y luego se enfrío a una temperatura inferior a la de reacción. En todos los casos, la mezcla del alimentación del reactor (caudal de 30 
$\mathrm{cm}^{3} \min ^{-1}$ ) fue $20,0 \%$ de $\mathrm{CH}_{4}, 2,0 \%$ de $\mathrm{N}_{2} \mathrm{O}$ y $78,0 \%$ de $\mathrm{He}$ [52]. Los resultados más relevantes se encuentran en la Tabla 5 , en la cual se observa que la mayor selectivi- dad para metanol fue $31,0 \%$ en mol con un valor de $0,65 \%$ en mol de conversión de metano cuando la temperatura de reacción fue $275^{\circ} \mathrm{C}$.

Tabla 5. Oxidación de metano a metanol

\begin{tabular}{|l|l|l|l|l|}
\hline Catalizador & Temperatura (K) & \multicolumn{1}{|c|}{$\begin{array}{c}\mathbf{H}_{2} \mathbf{O} \text { en la } \\
\text { alimentación } \\
(\%)\end{array}$} & $\begin{array}{c}\text { Conversión de } \mathbf{C H}_{4} \\
(\% \text { en mol) }\end{array}$ & $\begin{array}{c}\text { Selectividad } \mathrm{CH}_{3} \mathrm{OH} \\
(\% \text { en mol) }\end{array}$ \\
\hline \multirow{5}{*}{ FeZSM-5 } & 300 & 0,0 & 3,6 & 1,9 \\
\cline { 2 - 5 } & 300 & 1,0 & 1,9 & 9,3 \\
\cline { 2 - 5 } & 275 & 0,0 & 2,2 & 2,7 \\
\cline { 2 - 5 } & 275 & 1,0 & 0,65 & 31,0 \\
\hline
\end{tabular}

Fuente: elaboración propia, a partir de [53].

Los resultados de la Tabla 5 muestran cómo la temperatura afecta la conversión. La mayor conversión de metano se observa a la mayor temperatura, en cuanto a la selectividad ocurre el proceso contrario; la mayor selectividad a metanol se observa a la temperatura inferior. Otro aspecto es la introducción de agua en el reactor, la cual genera una mayor selectividad a metanol y esto disminuye la formación de coque [52].

En un estudio más reciente [53], se evaluó la actividad catalítica de cuatro tipos de zeolitas (Co/ZSM-5) en la oxidación parcial de metano y se observó el efecto del tamaño de poro y especies de cobalto en la reacción; asimismo, se estudiaron dos tipos de estructuras de zeolita (ZSM-5): micro poroso y mesoporoso, y dos formas de carga de Co: impregnación e intercambio iónico fueron estudiadas.

En [53] se destaca que el metano es un compuesto estable y en este proceso de conversión la activación del enlace $\mathrm{C}$-H del metano es considerada como el paso decisivo en la reacción catalítica teórica y experimentalmente; además, el uso del catalizador microporoso Mi_Co-ZSM-5 arroja el menor valor de conversión, $16,0 \%$ en mol. Esto se da porque el tamaño de los poros es muy pequeño y, por lo tanto, el transporte de los reactivos y productos es un proceso muy difícil [53].

Por otro lado, los catalizadores obtenidos por intercambio iónico y con un tamaño superior de poro, Hie_Co/ZSM, generaron una conversión del 7,56\% en mol de metano. El método de preparación permitió la formación de óxidos de cobalto que actúan como componentes activos del catalizador [53]. Desafortunadamente, en este estudio, los investigadores no presentaron los datos de selectividad.

Con base en la revisión de estos materiales, en la Fig. 5 se presentan los resultados de conversión de metano y selectividad a metanol expresados en porcentaje en moles. 


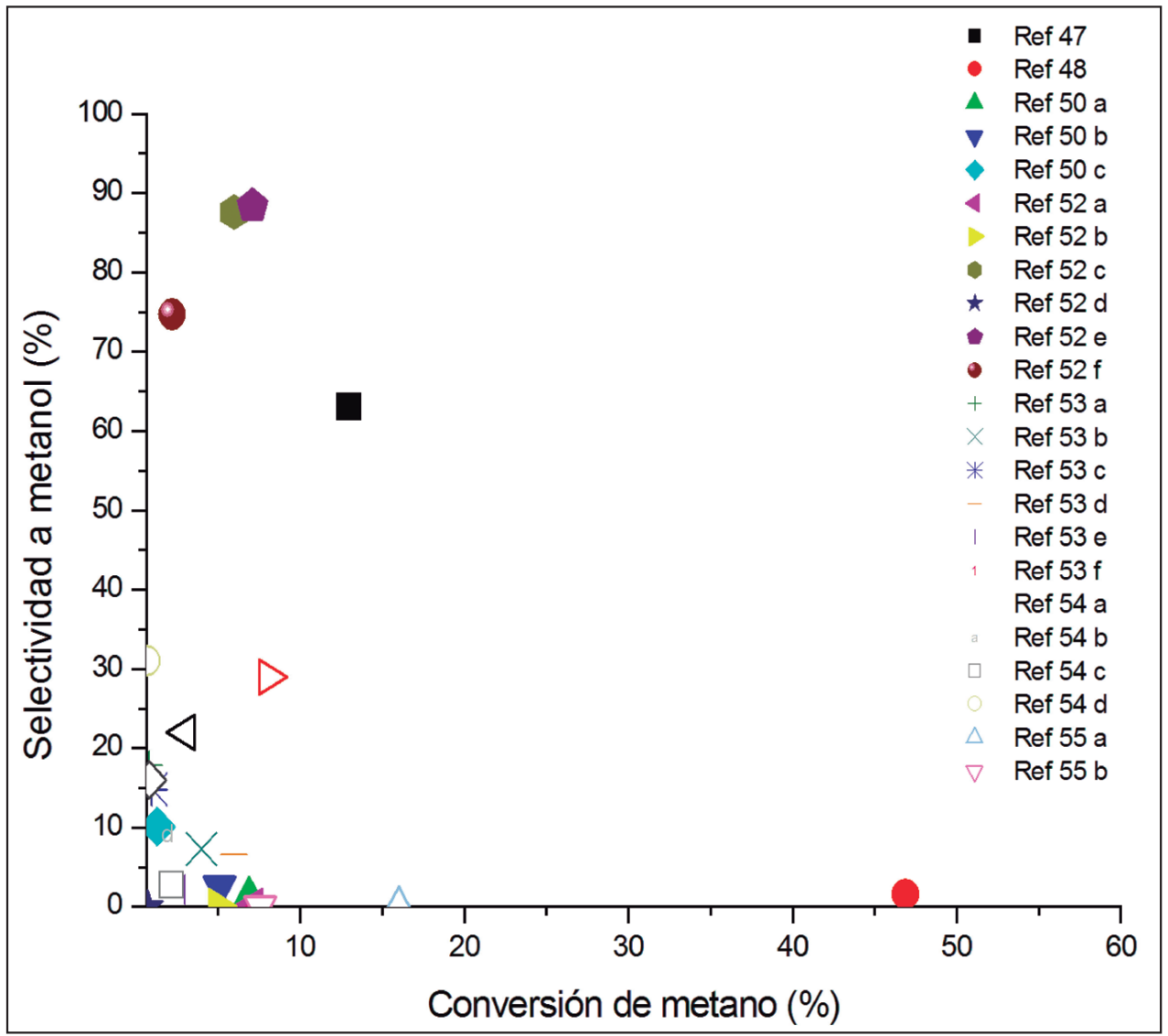

Fig. 5. Datos experimentales para la conversión de metano a metanol Fuente: elaboración propia, a partir de [46]-[47],[49], [51]-[54].

Como puede verse en la Fig. 5, la tendencia está dada por valores inferiores al $50,0 \%$ en mol de conversión de metano y valores de selectividad a metanol muy variados. En cuanto a la selectividad, hay valores altos, pero a bajas conversiones de metano. Se destaca como mayor valor de conversión de metano $46,9 \%$ en mol y como mayor valor de selectividad $88,4 \%$ en mol; sin embargo, el mayor valor de rendimiento de todos los ensayos realizados fue $8,19 \%$, lo cual muestra la gran dificultad de controlar un proceso basado en los aspectos mencionados previamente: 1) la dificultad de activar el metano; 2) evitar reacciones competitivas, y 3 ) una vez formado el metanol evitar que este se oxide y disminuir así el rendimiento del proceso.

\section{CONCLUSIONES}

De acuerdo con la revisión hecha, para llevar a cabo el proceso de oxidación selectiva de metano a metanol se deben evaluar diferentes condiciones para la síntesis de materiales catalíticos, teniendo en cuenta los siguientes factores: 1) el tipo de soporte; 2) componente activo (metal, óxido del metal); 3) energía de enlace entre los componentes del catalizador, reactivos y 
productos; 4) cantidad de carga el componente activo; 5) tiempo de envejecimiento, y 6) temperatura de calcinación.

Mientras que para el proceso de obtención del alcohol de manera directa, las variables por considerar son: 1) proporción de los gases de alimentación, 2) material del reactor; 3) velocidad espacial horaria; 4) temperatura de reacción, y 5) presión de trabajo.

Es importante tener presente que dentro de las variables de interés en el proceso se encuentra la energía de enlace entre los componentes del material catalítico, los reactivos y productos, ya que permitirá la adsorción de reactivos en la superficie del catalizador para promover la formación del producto de interés, en este caso el metanol.

De acuerdo con lo presentado y con base en algunos resultados obtenidos dentro de nuestro grupo de trabajo en el proceso de oxidación selectiva de metano a formaldehido -de los cuales se destacan conversiones de metano de $7,3 \%$ y $8,6 \%$ en mol y selectividad a formaldehído de $47,5 \%$ y $50,6 \%$ en mol cuando se trabajó a $650^{\circ} \mathrm{C}$ empleando como catalizador hierro soportado en óxido de silicio con una carga de $0,5 \%$ en masa [54], se evidenció que en reacciones de oxidación selectiva la carga del metal en el material catalítico tiene un papel importante en el rendimiento del proceso, debido a que una gran disponibilidad de sitios activos favorecerá la formación de óxidos inorgánicos.

Por otra parte, la estructura de poro de la sílice, cuando se prepara usando alquilsilicatos como precursores, es determinada principalmente por el tamaño y la geometría del empaquetamiento de las partículas de sílice [55]. Este tamaño permitirá la difusión, tanto de los reactantes, como productos dentro del material catalítico, lo cual favorece la formación de productos intermedios de oxidación como el metanol.

De acuerdo con las diferentes técnicas para la preparación de catalizadores, el procedimiento sol-gel permite obtener altas áreas superficiales con distribución de tamaños de poros alrededor de $4 \mathrm{~nm}$, a través de precursores metalorgánicos que se hidrolizan controlando el $\mathrm{pH}$ y la temperatura de la reacción de condensación [56]. De esta manera, se brindan características físicas favorables para que se lleve a cabo el proceso de oxidación selectiva de metano a metanol o formaldehído.

Por último, es importante seleccionar adecuadamente el soporte del material catalítico, ya que este influirá en el rendimiento del catalizador; particularmente, la sílice presenta mejor actividad que el soporte de titania en reacciones de oxidación parcial, ya que permite la dispersión de las especies de hierro, lo cual incide en la oxidación selectiva hasta formaldehído [56].

\section{AGRADECIMIENTOS}

Los autores agradecen al Departamento de Ingeniería Mecánica de la Universidad Nacional; al departamento de Química de la Universidad El Bosque; al departamento de Química de la Universidad Nacional, y al grupo de investigación Aprovechamiento Energético de Recursos Naturales (Aprena). 


\section{REFERENCIAS}

[1] J. Hargreaves, G. Hutchings y R. Joyner, "Control of product selectivity in the partial oxidation of methane," $\mathrm{Na}$ ture, vol. 348, pp. 428-429, 1990. DOI: https://doi.org/10.1038/348428a0

[2] R. Herman, Q. Sun, C. Shi y K. Klier, "Development of active oxide catalysts for the direct oxidation of methane to formaldehyde," Catalysis Today, vol. 37, no. 1, pp. 1-14, 1997. DOI: https://doi. org/10.1016/S0920-5861(96)00256-8

[3] Y. I. Pyatnitskii, "Contemporary methods for the direct catalytic conversion of methane," Theoretical and Experimental Chemistry, vol. 39, no. 4, pp. 201-218, 2003. DOI: https://doi.org/10.1023/A:1025797710504

[4] K. Fujimoto, F. H. Ribeiro, F. M. Avalos-Borja y E. Iglesia, "Structure and Reactivity of $\mathrm{PdO}_{x} / \mathrm{ZrO}_{2}$ Catalysts for Methane Oxidation at Low Temperatures," Journal of Catalysis, vol. 179, no. 2, pp. 431-442, 1998. DOI: https:// doi.org/10.1006/jcat.1998.2178

[5] Bp., "Natural gas reserves," 2016. [En línea]. Disponible en: http:// www.bp.com/en/global/corporate/ energy-economics/statistical-review-of-world-energy/natural-gas/natural-gas-reserves.html

[6] C. J. Castaneda, "Historical Overview of the Natural Gas Industry," Reference Module in Earth Systems and Environmental Sciences, pp. 1-11, 2014. DOI: https://doi.org/10.1016/b978-0-12409548-9.09034-5
[7] G. Rasul, G. K. S. Prakash y G. A. Olah, "Comparative study of the hypercoordinate carbonium ions and their boron analogs: A challenge for spectroscopists," Chemical Physics Letters, vol. 517, no. 1-3, pp. 1-8, 2011. DOI: https:// doi.org/10.1016/j.cplett.2011.10.020

[8] J. March. Advance Organic Chemistry: Reactions, Mechanisms and Structure. Nueva York, Estados Unidos: McGraw-Hill Book Company, 1968.

[9] H. Schwarz, "Chemistry with Methane: Concepts Rather than Recipes," Angewandte Chemie International Edition, vol. 50, no. 43, pp. 10096-10115, 2011. DOI: https://doi.org/10.1002/ anie.201006424

[10] T. J. Hall, J. S. J. Hargreaves, G. J. Hutchings, R. W. Joyner y S. H. Taylor, "Catalytic synthesis of methanol and formaldehyde by partial oxidation of methane," Fuel Process. Technol., vol. 42, no. 2-3, pp. 151-178, 1995. DOl: https:// doi.org/10.1016/0378-3820(94)00125-d

[11] P. Khirsariya y R. K. Mewada, "Single Step Oxidation of Methane to MethanolTowards Better Understanding," Procedia Engineering, vol. 51, pp. 409-415, 2013. DOI: https://doi.org/10.1016/j. proeng.2013.01.057

[12] P. Aghalayam, Y. K. Park, N. Fernandes, V. Papavassiliou et al., "A C1 mechanism for methane oxidation on platinum," Journal of Catalysis, vol. 213, no. 1, pp. 2338, 2013. DOI: https://doi.org/10.1016/ S0021-9517(02)00045-3 
[13] Q. Zhang, D. He y Q. Zhu, "Recent Progress in Direct Partial Oxidation of Methane to Methanol," Journal of Natural Gas Chemistry, vol. 12, pp. 81-89.

[14] Methanol Institute, "Methanol producction," 2013. [En línea]. Disponible en: http://www.methanol.org/the-methanol-industry

[15] N. R. Foster, “Direct Catalytic Oxidation of Methane to Methanol - A Review," Appl. Catal., vol. 19, no. 1, pp. 1-11, 1985. DOI: https://doi.org/10.1016/ S0166-9834(00)82665-2

[16] D. Lance y E.G. Elworthy, "Process for the manufacture of Methyl-alcohol from Methane," British patent no. 7297, Jan. 17,1907.

[17] R. T. Elworthy, “Can. Dept. Mines, Summary Report," no. 586, 1921.

[18] B. K. Hodnett. Heterogeneous Catalytic Oxidation. London, United Kingdom: John Wiley \& Sons Inc., 2000.

[19] M. J. da Silva, "Synthesis of methanol from methane: Challenges and advances on the multi-step (syngas) and one-step routes (DMTM)," Fuel Processing Technology Journal, vol. 145, pp. 42-61, 2016. DOI: https://doi. org/10.1016/j.fuproc.2016.01.023

[20] V. I. Atroschchenko, N. A. Gavrya, and Z. M. Shchedrinskaya, USSR Patent no. SU 132623 A1 1960.

[21] V. I. Atroshchenko, Z. M. Shchedrinskaya y N. A. Gavrya, "Catalysts for the oxidation of natural gas to formaldehyde and methanol," Zhurnal Prikladnoi Khimii, vol. 38, no. 3, pp. 643-649, 1965.
[22] V. I. Atroshchenko y Z. M. Shchedrinskaya, "Inst". Tr.Khark. Politekhn. Inst., vol. 39, no. 19, 1962.

[23] L. I. Avramenko y R. V. Kolesnikova, "Reactions of the simplest hydrocarbons with atomic oxygen (in Russian)," Izd. ANSSSR, Moscow, 1954.

[24] I. A. Makarov, USSR Patent no. 205000, 1967.

[25] M. Eusuf, Khursheed-ul-Islam, y $M$. Moslem, Sci. Res.(Dacca), vol. 5, no. 2-3, p. 104, 1968.

[26] M. Eusuf y M. Ahmed, Bangladesh J.Sci. Ind., vol. 6, no. 1-2, p. 46, 1969.

[27] R. S. Mann y M. K. Dosi, "Partial oxidation of methane to formaldehyde over halogen modified catalyst," Journal of Chemical Technology and Biotechnology, vol. 29, pp. 467-469, 1979. DOI: https:// doi.org/10.1002/jctb.503290803

[28] A. Y. Averbuch, I. P. Mukhlenov, y Y. A. Ivanov, Prevrashch. Uglevodorodov Kislotno - Osnovn. Geterogennykh Katal, 1977.

[29] D. A. Dowden y G. T. Walker, British Patent no. 1244001, 1971.

[30] H. J. F. Stroud, British Patent no. $1398385,1975$.

[31] V. K. Leonov, I. A. Ryzhak, L. M. Kalinichenko, Y. L. Vysotskii, L. E. Semikina, y Y. A. Shuster, Katal. Katal, vol. 15, p. 6, 1977.

[32] R. S. Liu, M. Iwamoto y J. H. Lunsford, "Partial oxidation of methane by nitrous oxide over molybdenum oxide supported on silica," Journal of the 
Chemical Society, Chemical Communications. vol. 1, p. 78, 1982. DOI: https:// doi.org/10.1039/c39820000078

[33] M. Iwamoto, Japanese Patent no. 5892630, 1983.

[34] M. Iwamoto, Japanese Patent no. 5892629, 1983.

[35] H. D. Gesser, N. R. Hunter, y L. A. Morton, International Chemical Congress of Pacific Basin Societies, 1984.

[36] N. R. Hunter, H. D. Gesser, L. A. Morton y P. S. Yarlagadda, "Methanol Formation at High Pressure by the Catalyzed Oxidation of Natural Gas and by the Sensitized Oxidation of Methane," Applied Catalysis, vol. 57, no. 1, pp. 45-54, 1990. DOI: https://doi.org/10.1016/ S0166-9834(00)80722-8

[37] N. D. Spencer, C. J. Pereira y R. K. Grassellit, "The Effect of Sodium on the MoO3-SiO2 - Catalyzed Partial Oxidation of Methane," Journal of Catalysis, vol. 126, no. 2, pp. 546-554, 1990. DOI: https://doi.org/10.1016/00219517(90)90019-G

[38] J. E. Lyons, P. E. Ellis y V. A. Durante, "Active iron oxo centers for the selective catalytic oxidation of Alkanes," Studies in Surface Science and Catalysis, vol. 67, pp. 99-116, 1991. DOI: https://doi. org/10.1016/s0167-2991(08)61930-8

[39] Q. Sun, R. Herman y K. Klier, "Selective oxidation of methane with air over silica catalysts," Catalysis Letters, vol. 16, no. 3, pp. 251-261, 1992. DOI: https:// doi.org/10.1007/BF00764337
[40] G. S. Walker, J. A. Lapszewicz y G. A. Foulds, "Partial oxidation of methane to methanol - comparison of heterogeneous catalyst and homogeneous gas phase reactions," Catalysis Today, vol. 21, no. 2-3, pp. 519-526, 1994. DOI: https://doi.org/10.1016/09205861(94)80175-4

[41] T. J. Hall, J. S. J. Hargreaves, G. J. Hutchings, R. W. Joyner y S. H. Taylor, "Catalytic synthesis of methanol and formaldehyde by partial oxidation of methane," Fuel Processing Technology, vol. 42, no. 2-3, pp. 151-178, 1995. DOI: https://doi.org/10.1016/03783820(94)00125-D

[42] R. Raja y P. Ratnasamy, "Direct conversion of methane to methanol," $A p$ plied Catalysis a-General, vol. 158, no. 1-2, pp. L7-L15, 1997. DOI: https://doi. org/10.1016/S0926-860X(97)00105-1

[43] S. H. Taylor, J. S. J.Hargreaves, G. J. Hutchings, R. W. Joyner y C. W. Lembacher, "The partial oxidation of methane to methanol: An approach to catalyst design," Catalysis Today, vol. 42, no. 3, pp. 217-224, 1998. DOI: https://doi.org/10.1016/S09205861(98)00095-9

[44] R. L. McCormick, M. B. Al-sahali y G. O. Alptekin, "Partial oxidation of methane, methanol, formaldehyde, and carbon monoxide over silica: global reaction kinetics," Applied Catalysis A: General, vol. 226, no. 1-2, pp. 129-138, 2002, DOI: https://doi.org/10.1016/ S0926-860X(01)00894-8 
[45] Q. Zhang, D. He, J. Li, J., B. Xu et al., "Comparatively high yield methanol production from gas phase partial oxidation of methane," Applied Catalysis A: General, vol. 224, no. 1-2, pp. 201-207, 2002. DOI: https://doi.org/10.1016/ S0926-860X(01)00820-1

[46] L. Chen, X. Zhang, L. Huang y L. Lei, "Application of in-plasma catalysis and post-plasma catalysis for methane partial oxidation to methanol over a Fe203-CuO/Y-Al2O3 catalyst," Journal of Natural Gas Chemistry, vol. 19, no. 6, pp. 628-637, 2010. DOI: https://doi. org/10.1016/S1003-9953(09)60129-8

[47] N. V. Beznis, A. N. C. van Laak, B. M. Weckhuysen y J. H. Bitter, "Oxidation of methane to methanol and formaldehyde over Co-ZSM-5 molecular sieves: Tuning the reactivity and selectivity by alkaline and acid treatments of the zeolite ZSM-5 agglomerates," Microporous and Mesoporous Materials, vol. 138 , no. $1-3$, pp. 176-183, 2011. DOI: https://doi.org/10.1016/j.micromeso.2010.09.009

[48] R. Polnišer, M. Štolcová, M., Hronec y M.Mikula, "Structure and reactivity of copper iron pyrophosphate catalysts for selective oxidation of methane to formaldehyde and methanol," Applied Catalysis A: General, vol. 400, no. 1-2, pp. 122-130, 2011. DOI: https://doi. org/10.1016/j.apcata.2011.04.022

[49] F. Xu, L. Zhu, F. Hou y B. Shen, "Selective oxidation of methane to methanol in acetic acid solvent with $\mathrm{Pd}(\mathrm{OAc}) 2$-p-benzoquinone-CO catalyst system," Jour- nal of Fuel Chemistry and Technology, vol. 40, no. 9, pp. 1098-1102, 2012. DOI: https://doi.org/10.1016/S18725813(12)60040-1

[50] Y. Hu, M. Anpo y C. Wei, "Effect of the local structures of $\mathrm{V}$-oxides in MCM-41 on the photocatalytic properties for the partial oxidation of methane to methanol," Journal of Photochemistry and Photobiology A: Chemistry, vol. 264, pp. 48-55, 2013. DOI: https://doi.org/10.1016/j.jphotochem.2013.05.005

[51] S. Mansouri, O. Benlounes, C. Rabia, R. Thouvenot et al., "Partial oxidation of methane over modified Keggin-type polyoxotungstates," Journal of Molecular Catalysis A: Chemical, vol. 379, pp. 255-262, 2013. DOI: https://doi. org/10.1016/j.molcata.2013.08.006

[52] M. V. Parfenov, E. V. Starokon, L. V. Pirutko y G. I. Panov, "Quasicatalytic and catalytic oxidation of methane to methanol by nitrous oxide over FeZSM-5 zeolite," Journal of Catalysis, vol. 318, pp. 14-21, 2014. DOI: https:// doi.org/10.1016/j.jcat.2014.07.009

[53] Y. Krisyuningsih Krisnandi, B. Ary Pujangga Putra, M. Bahtiar, I. Abdullah y R. Francis Howe, "Partial Oxidation of Methane to Methanol over Heterogeneous Catalyst Co/ZSM-5," Procedia Chemistry, vol. 14, pp. 508-515, 2015. DOI: https://doi.org/10.1016/j. proche.2015.03.068

[54] C. A. Guerrero-Fajardo, D. Niznansky, Y. N'Guyen, C. Courson y A.-C- Roger, "Methane selective oxidation to formal- 
dehyde with Fe-catalysts supported on silica or incorporated into the support," Catalysis Communications, vol. 9, no. 5, pp. 864-869, 2008. DOI: https://doi. org/10.1016/j.catcom.2007.09.013

[55] C. A. Guerrero-Fajardo, Y. N'Guyen, C. Courson y A.-C. Roger, "Catalizadores $\mathrm{Fe} \mathrm{SiO} 2$ para la oxidación selectiva de metano hasta formaldehido," Inge- niería E Investigación, vol. 26, no. 2, pp. 37-44, 2006.

[56] C. A. Guerrero-Fajardo, J. F. Castellanos, A.-C. Roger y C. Courson, "Síntesis sol-gel de catalizadores de hierro soportados sobre sílice y titania para la oxidación selectiva de metano hasta formaldehído," Ingeniería e Investigación, vol. 28, no. 1, pp. 72-80, 2008. 